\title{
Claves para la comprensión de una Historia Estelar: interpretación, comprensión, hermenéutica y diversidad política ${ }^{1}$
}

Robert Ojeda Pérez ${ }^{2}$

Universidad La Salle, Bogotá, Colombia ${ }^{3}$

robert.rojeda@gmail.com

1 Artículo de Investigación.

2 Magíster en Historia, Universidad de Los Andes; Candidato a Ph.D Universidad Pedagógica Nacional, Bogotá, Colombia.

3 Docente tiempo completo. 


\title{
Claves para la comprensión de una Historia Estelar: interpretación, comprensión, hermenéutica y diversidad política
}

\section{Resumen}

Al revisar la historiografía se pueden apreciar los distintos enfoques con los que se ha divulgado y enseñado la historia en este país. Tanto la investigación como la enseñanza de la historia requieren de una nueva manera de interpretar y comprender la realidad social en la que se presente la intención discursiva del emisor y del investigador. En este artículo se hace énfasis en la intención política de la historia al querer promulgar la memoria de una sociedad. Se busca que a la hora de investigar y de enseñar la historia se tengan en cuenta a los distintos actores de una comunidad con el objetivo de poder construir nuevas dinámicas de ciudadanía participativa desde un lugar plural de convivencia.

Palabras clave: Educación, enseñanza de la historia, diversidad, comprensión, Historia Estelar.

\section{Keys for understanding a Stellar History: interpretation, understanding, hermeneutics and political diversity}

\begin{abstract}
When reviewing the historiography of Colombia one can appreciate the different approaches with which history has been disclosed and taught. In that review, it's revealed that both research and teaching of history require a new way to interpret and comprehend the social reality, on which the discursive intention of the speaker and the researcher are presented. In this article the political intention of history is analyzed when it wants to promote the memory of a society. That being so, it's sought that the different actors of a community are taking into account when researching and teaching history, in order of being able to build new dynamics of participative citizenry from a common place of plural coexistence
\end{abstract}

Keywords: Education, teaching of history, diversity, understanding, stellar history.

\section{Propostas para o entendimento de uma história Estelar: interpretação, entendimento, her- menêutica e diversidade política.}

\section{Resumo:}

Ao revisar a historiografia, podem ser apreciados os diferentes enfoques com os que se divulgou e ensinou história neste país. Tanto a investigação, como o ensino da história, requer uma nova maneira de interpretar e compreender a realidade social na que se apresente a intenção discursiva do emissor e do pesquisador. Neste artigo, faz-se ênfase na intenção política da história, ao querer promulgar a memória de uma sociedade. Procura-se, que na hora de pesquisar e de ensinar a história, se tenham em conta os diferentes atores de uma comunidade com o objetivo de poder construir novas dinâmicas de cidadania participativa desde um lugar plural de convivência.

Palavras-chave: Educação, ensino da história, diversidade, entendimento, história Estelar. 


\section{Introducción}

El modelo historiográfico heredado del siglo xIx concebía la historia como una serie de acontecimientos que estaban plasmados en los documentos escritos manuscritos o impresos- Sin embargo, ello cambia tras la aparición de distintas escuelas de pensamiento histórico - como la Escuela de los Annales en Francia- y las discusiones hermenéuticas.

Es a la luz de estos nuevos enfoques donde se sitúa la discusión teórica y metodológica de este escrito, el cual mostrará que en la recuperación e interpretación del pasado se requiere de unos pasos previos que se deben tener en cuenta: la interpretación, la hermenéutica, la comprensión o la diversidad política del enfoque. Este artículo aborda la discusión de las fuentes documentales para la investigación histórica y hace una propuesta para la enseñanza de la historia, teniendo en cuenta un marco teórico de referencia como la hermenéutica, con el fin de construir y repensar otras dinámicas de ciudadanía.

Para poder hablar del problema de las fuentes en el desarrollo de la historia se tendrán en cuenta los siguientes puntos: primero, el uso de las fuentes primarias en la formulación de un acontecimiento; segundo, el papel de la historia como herramienta política; y, por último, un nuevo enfoque para enseñar la historia y dejar atrás la crisis de un país en guerra. En cuanto a la propuesta de enseñanza de la historia se propone aquí un modelo titulado por el autor como la Historia Estelar, el cual parte del círculo hermenéutico que se abordará más adelante.

El objetivo es hacer una reflexión tanto de la investigación histórica como de la enseñanza y difusión de la historia. Para ello se quiere recalcar la importancia de la formación de ciudadanos críticos en la reconstrucción de la memoria y de la identidad nacional a partir de la comprensión del pasado. Se hará, por consiguiente, un recorrido que comienza por las experiencias en dos campos a saber: el de la investigación y el de la didáctica de la historia.

\section{Hecho histórico versus acontecimiento}

Uno de los historiadores más representativos del siglo xx se refiere al hecho histórico como una construcción realizada por el historiador, «inventado y fabricado mediante hipótesis y conjeturas, a través de un trabajo delicado y apasionante (...) Elaborar un hecho significa construirlo. Si se quiere, proporcionar la respuesta a un problema» (Febvre 1959; 1982, 38-39; Herrera, 2009). En esta medida los nuevos enfoques de la historia no consisten en enumerar las causas y las consecuencias de los hechos ni estar centrados en la objetividad o veracidad de los procesos con pretensiones de verdad. 
Por otro lado, hay que tener en cuenta que cuando se pretende reconstruir un hecho histórico, más allá del ejercicio de comprensión y contextualización, la pregunta que se puede hacer un ciudadano es: ¿puedo ser parte activa de la interpretación del pasado para comprender el presente? ¿Puedo trascender la sociedad en la que vivo?

Herrera expone que: «tras entender el hecho histórico como una construcción desde una "historia problema" y redimensionar las nociones propias de objetividad y verdad, la ciencia histórica otorga un nuevo lugar a la noción de interpretación» (Herrera 2009, 57). La historia tiene un gran componente narrativo producto de la interpretación de los acontecimientos, de la lectura simbólica de los mismos, puestos ordenadamente bajo un hilo conductor o trama por el historiador. Si la historia es un discurso no acabado, ¿cuáles deben ser considerados como acontecimientos históricos? Los historiadores reflexionan sobre el presente y el futuro, lo cual alimenta nuevas lecturas sobre el pasado y cómo ellos pueden ser partícipes, pero entonces, ¿cuál es el papel de la memoria?

La memoria es producto de una relación dialéctica entre el recuerdo y el olvido. Es una supresión de elementos para poder conservar otros en la medida en que nos es imposible recordarlo todo. Por consiguiente, la memoria resulta de una selección (Zapata 2004, 10). Forzosamente unos elementos son conservados para que otros sean progresivamente marginados hasta que se olviden del todo (Todorov 2000, 15-16)

En ese proceso de selección de lo que se quiere recordar juega un papel fundamental el presente (Ricoeur 2003, 526) ya que la acción de seleccionar está condicionada por el presente en la medida en que se le da un uso en la actualidad a lo seleccionado. Esto igualmente pasa con lo olvidado, a la que no se le reconoce utilidad en el presente (Barbosa 2001, 104-112).

En este sentido la memoria es una operación simbólica pues la narración en la que se constituye la memoria, al rescatar la narración desde el presente, no son relatos factuales de hechos escuetos, sino relatos de construcción de identidad: «las memorias documentan una manera de pensar propia de un grupo en un momento determinado» (Barbosa 2001, 108). La correlación entre memoria y olvido se conecta con la posibilidad de hacer consciente el recuerdo dejado en el olvido por percepciones sensoriales inesperadas (Zapata, 2004, 9). Este hecho se ilumina de una forma mucho más clara con las nociones de memoria presentadas por Marcel Proust en su obra En busca del tiempo perdido (Proust 2000) y en las reflexiones que sobre el tema hace el filósofo y crítico literario alemán Walter Benjamin (Benjamin 2001, 125-132).

Las preguntas que un historiador se hace en su presente y transfiere al pasado son distintas en cada momento y dependen de diversos enfoques, intereses y problemas que se quieren resolver en la comprensión de la sociedad desde la actualidad. De 
Claves para la comprensión de una Historia Estelar: interpretación, comprensión, hermenéutica y diversidad política

esta manera hay que tener en cuenta que las dinámicas sociales y humanas son distintas y cambiantes con el paso del tiempo. En ese orden de ideas, la sociedad y los historiadores pueden permitirse olvidar en algunos momentos algunos pasajes de la reconstrucción del pasado; pueden reescribir los acontecimientos no como hechos históricos inmutables, sino como «acontecimientos» que pueden ser interpretados de distintas maneras $y$, asimismo, pueden ser narrados desde otros enfoques que permitan una mayor comprensión de los mismos.

A propósito de la comprensión, siguiendo a Hannah Arendt, para comprender una sociedad múltiple se necesita una comprensión que sea capaz de ver sus diferentes rasgos, que sea diversa y mutable, para poder sentirnos en armonía con el mundo; sin embargo, para Arendt entender al mundo no es algo activo que contenga dentro de sí algún tipo de movimiento. Sólo a partir de la reflexión, que llega a generar algún movimiento, «la comprensión no tiene fin y por lo tanto no puede producir resultados definitivos» (Arendt, 1995: 30).

Bien señala Herrera en su juiciosa lectura de Le Goff:

La intervención del futuro en las reinterpretaciones de la historia se relaciona directamente con los métodos y técnicas de trabajo del historiador. El reto que enfrentan los historiadores para construir problemas y hechos históricos pasa por la permanente vigilancia a los conceptos y esquemas con los que regularmente operan... (Herrera, 2009: 59).

Lo que se quiere enfatizar aquí es que a medida que ha pasado el tiempo los intereses de consulta y las preguntas a los documentos han cambiado; no siempre se utilizan los mismos documentos o, en ocasiones, sí se usan los mismos, pero se les realizan otras preguntas y se establecen otros problemas, lo cual hace que los historiadores acudan algunas veces a la misma fuente pero con otras preocupaciones de investigación o, por el contrario, se consulten otras fuentes bajo otros marcos de interpretación.

Por ejemplo, en el siglo xvIII la descripción en las fuentes y en los documentos era casi poética y extremadamente literaria. Los investigadores utilizaron esas fuentes en el Siglo de las Luces para hacer unas descripciones de tipo costumbrista que se impusieron a comienzos del siglo xIx. Es el caso del libro El pueblo de Jules Michelet, en el que las preocupaciones eran otras, por tanto, el uso de las fuentes fue distinta y eso obliga a que el investigador deba seleccionar el material de trabajo.

Ya en pleno siglo xix y por influencia de Ranke, uno de los impulsores del positivismo en la investigación histórica, se motivaba a que los trabajos estuvieran cargados de una búsqueda exhaustiva de la verdad en el documento; por tal motivo, los investigadores de esa época debían interpretar el hecho histórico como una 
realidad aséptica y objetiva. Señalaban que para hacer historia bastaba con dejarse llevar por los documentos y se renunciaba a la interpretación.

Por el contrario, para el siglo xx los historiadores bebieron de otros modelos de las ciencias sociales. Incorporaron nuevos enfoques, implementaron las preguntas a las fuentes, utilizaron la hermenéutica como posibilidad de comprender la realidad, lo cual desarrolló una nueva forma de hacer historia como, por ejemplo, el uso e interpretación de las fuentes para reconstruir un pasado. Esta reconstrucción pudo ser fragmentaria debido a que los que registraron estos hechos tenían un interés particular ya fuera para defender, mostrar u ocultar.

En esa medida, la acción del historiador como docente busca que sus estudiantes profundicen la historia desde el uso de las fuentes primarias, debe contemplar los distintos enfoques, corrientes y escuelas historiográficas para poder orientar el trabajo de la búsqueda en los archivos para resolver preguntas y problemas. Como decía Le Goff: «la historia es la ciencia del pasado, con la condición de saber que este se convierte en objeto de la historia a través de una reconstrucción que se pone en cuestión continuamente» (Le Goff 1991, 39).

Hay que tener en cuenta que los registros pasan por otras manos que ciernen y alejan a los lectores de la comprensión e interpretación del pasado, que éstos no pueden ser universales ni totalizantes, que no puede haber una única versión del mismo, sino más bien interpretaciones en las que caben distintos argumentos desde la hermenéutica. Por eso el papel fundamental de esta última en cuanto teoría.

La hermenéutica en la antigüedad clásica fue un ejercicio riguroso de lectura por el cual se encontraron los medios para "poner a distancia» la subjetividad invasora que quería decir al texto sólo lo que ella buscaba, sin respetar toda su riqueza. Junto a esa visión clásica de la hermenéutica como ciencia que aporta reglas de interpretación correcta de los textos existe hoy otra más amplia y densa: la que acentúa la naturaleza histórica y lingüística de nuestra experiencia del mundo, de nuestro conocimiento (Siciliani 2013).

\section{De la hermenéutica a la Historia Estelar}

Para realizar el proyecto de la Historia Estelar tomaremos como antecedente teórico a la Hermenéutica cuyo punto central es la comprensión a través de la interpretación, la cual está basada en un proceso abierto que ninguna visión puede finalizar el círculo de interpretación-. Como la intuición hace parte de la investigación histórica, por tanto, requiere de interpretación. Según Ricoeur:

La interpretación tiene connotaciones subjetivas específicas, tales como la implicación del lector en el proceso de comprensión y la reciprocidad entre 
Claves para la comprensión de una Historia Estelar: interpretación, comprensión, hermenéutica y diversidad política

la interpretación del texto y la interpretación de sí mismo. Esta reciprocidad es conocida bajo el nombre del círculo hermenéutico; implica una oposición marcada a esa suerte de objetividad y de no implicación que se supone caracteriza a la explicación científica de las cosas. (Ricoeur 2008, 39)

Para analizar la función principal de la configuración narrativa o Historia Estelar, como se ha querido señalar desde este escrito, es necesario en primera instancia reflexionar acerca de la postura crítica de la noción de temporalidad presentada en el Libro xı de las Confesiones de san Agustín, para luego proseguir con la relación manifiesta entre Interpretación, comprensión y diversidad política, entendidas como: la estructura pre- narrativa de la acción, «prefiguración» — Interpretación-; la estructura del texto o entramado «configuración»—comprensión-; y, finalmente, la «aplicación» de la configuración de la pluralidad en la construcción de memoria, «refiguración» — diversidad política_- La Historia Estelar es, pues, un entramado simbólico que permite la comprensión del pasado; se puede comparar como una constelación de significados dentro de una función narrativa, interpretativa y comprensiva de la historia, que tiene implicaciones en la experiencia personal. Así entendidas, estas tres nociones, en su simultaneidad, nos llevarán a la comprensión de una historia — en este caso la historia de un individuo en su contexto—, además de presenciar la narratividad como elemento fundamental en la construcción de una trama — historia — de un individuo en la medida en que él narra su vida y, por ende, se comprende a sí mismo.

La percepción y el conocimiento de este primer acercamiento nos sugieren determinar el concepto ricoeuriano de historia a través de un largo viaje por el relato histórico que presenta así la estructurara de la experiencia y las fuentes que se emplean en su construcción, de ahí se formulará la hipótesis de que la identidad narrativa, sea de una persona o sea de una comunidad será el fin buscado de la nueva forma de hacer historia. En efecto, la construcción de la trama da un elemento más legible de las vidas humanas cuando éstas son interpretadas en función de las historias que la gente cuenta a propósito de ellos mismos. Estas historias de vida se hacen a su vez más inteligibles cuando se les aplican modelos narrativos, pequeñas tramas tomadas de las historias propiamente dichas (Ricoeur 2006, 106-107).

En consecuencia, en las anteriores aserciones se halla implícita la comprensión del sí mismo, por medio de la narración envuelta de signos y símbolos, como una mediación privilegiada. Tal mediación se vale tanto de la historia como de la narración de ficción haciendo de la historia de vida una narración de ficción o, si se prefiere, una ficción histórica (Ricoeur 2006, 107). Al entrecruzar el estilo historiográfico de una autobiografía con el estilo novelesco de un relato de ficción se pone de manifiesto el nuevo objeto de la historia. 
El problema de la identidad narrativa en la Historia Estelar, esta nueva forma de hacer historia, es una clara comprensión de que lo que está en juego es la cuestión misma de la identidad narrativa aplicada a una persona —individuo- o, si se quiere, a una nación.

\section{Papel de la historia como herramienta política}

La Historia, disciplina perteneciente a las ciencias humanas y sociales, tiene como objetivo primordial comprender el devenir de los seres humanos en el tiempo, es decir, en vez de juzgar el decurso histórico de la humanidad — premisa que durante décadas se consideró esencial para poder adelantar estudios en la materia-, lo que fundamentalmente pretende es interpretar de manera adecuada y coherente la realidad pasada, presente y futura de un determinado conglomerado social —sea entendido como pueblo, comunidad, o sociedad-.

Indudablemente, esta tarea implica la producción de un conocimiento que, siendo propio de la disciplina, se encuentra sin embargo claramente vinculado a los problemas epistemológicos de las demás ciencias humanas y sociales. Tal situación se explica si se tiene en cuenta que la totalidad de las disciplinas inscritas dentro de dicha área del saber comparten un mismo estatuto gnoseológico que es, precisamente, el que las dota de sentido. (Herrera 2009, 64). Es importante recalcar esa unidad para poder entender la especificidad de los estudios históricos, puesto que si bien es cierto que todas las ciencias humanas y sociales comparten el interés por indagar sobre los seres humanos, su naturaleza, sus actos y su relación con el entorno que los rodea, la historia se preocupa por la memoria, el recuerdo y el olvido, para reconstruir la identidad de los distintos grupos sociales.

En Colombia, desde principios del siglo xx, la práctica de la Historia había estado a cargo de amateurs, de "caballeros andantes de patriotismo» (Archila 1997, 173205) quienes asumían el papel de guardianes de la memoria oficial. Aparte de impulsar una conciencia nacionalista tan cara a nuestros proyectos de construcción del Estado-nación, su interés no cobijaba una preocupación por los métodos y teorías de la disciplina, constituyendo así una historia de aficionados para aficionados, una «historia homogeneizadora» bajo el amparo de la Academia Colombiana de Historia creada en 1902 (Bonilla 1997, 7-15)

Para la difusión de este tipo de historia, centrada en la historia militar y política, los manuales escolares jugaron un papel importante en la transmisión de un tipo de memoria histórica que ha sido caracterizada como «irreflexiva, conservadora, elitista y nacionalista» (Lewis 2003, 48) por el tipo de nación blanca, hispánica y católica que pregonaban. Este modelo de nación fue defendido por la Constitución de 1886, carta donde adquieren «forma institucional los pilares más importantes de una invención nacional homogeneizante» (Wills Obregón 2000, 385-415). 
Claves para la comprensión de una Historia Estelar: interpretación, comprensión, hermenéutica y diversidad política

¿Espejito, espejito, cuál es la historia oficial de Colombia? Así como se miraba la bruja malvada en el cuento de Blancanieves — sólo se miraba a sí misma sin querer aceptar las otras imágenes-Colombia se ha estado mirando al espejo desde una sola perspectiva. "El adoctrinamiento puede ser peligroso porque tiene su origen en una perversión, no del conocimiento, sino de la compresión» (Arendt 1995, 30) Por eso es importante que al enseñar la historia se relacione claramente quién es el agente de enunciación para que no se caigan en vicios de subjetividades ni pretensiones de objetividad. Se debe relacionar al sujeto cultural como agente de un discurso capaz de generar acciones.

Si el sentido de la política es la libertad, es en este espacio donde tenemos el derecho a esperar milagros. No porque creamos en ellos sino porque los hombres, en la medida en que pueden actuar, son capaces de llevar a cabo lo improbable y lo imprevisible y de llevarlo a cabo continuamente, lo sepan o no... (Arendt 1995, 66).

Por eso la importancia de la Historia Estelar, concepto que enmarca una multiplicidad de versiones, sujetos y actores que pueden ser rescatados desde distintas fuentes. En estos nuevos relatos, bajo la interpretación pero sobre todo bajo la narración, se pueden evidenciar las constelaciones que permiten establecer una comprensión del pasado.

Sin embargo, hoy la investigación histórica ha mostrado un avance importante aunque, como lo señala Jorge Orlando Melo, la abundancia de publicaciones «cubre un abanico temático cada vez más amplio, sobre todo en los historiadores más jóvenes» (Melo 1999, 20), mientras que por parte de los historiadores con trayectoria sus trabajos reflejan madurez en la escogencia de sus objetos de estudio como en los enfoques teóricos utilizados, «son una muestra de la vitalidad del trabajo histórico que se hace en el país». (Melo 1999, 21) No obstante, todavía ésta sigue siendo insular, no se proyecta como debería hacia la enseñanza básica y mucho menos hacia el público en general; todavía existe indisposición hacia la historia como una materia aburrida.

\section{Enfoque de la Historia como instrumento político}

La educación es un instrumento por medio del cual se pueden modelar sujetos para distintos fines. Hoy en día está circulando por la web un video en el cual se señala que la educación es productora de productores y está al servicio de la economía del mercado. Pues bien, la educación no se puede dejar de ver como un instrumento de poder, pero es muy distinto para qué se utiliza y con qué fin. A lo largo de la historia han existido instituciones que se preocupan por el librepensamiento, maestros que lo promueven, así como instituciones que regulan, controlan y normativizan hasta las formas de hablar, sentir, comunicar y liderar: 
Pero no sólo instituciones educativas, sino también instituciones de poder gubernamental y hasta estatal. Pensemos por unos momentos la producción de conocimiento por parte de los monasterios medievales, en donde se estipulaba qué era lo que se pensaba, hacía y comunicaba. En la época del enciclopedismo y la Revolución francesa se promulgaron los derechos del hombre y del ciudadano, pero seguido a ello la igualdad, que fue mal entendida y desarrollada pues por el afán de reconocer a los otros ante el derecho se despreció la capacidad de deliberación, la capacidad de participación en las leyes. En el siglo xx se habla de totalitarismo, de imperialismo, y el conocimiento es desarrollado desde academias reales de la ciencia, de la lengua y de la historia. Pensemos por un momento en la Enciclopedia Británica y en las instituciones que han querido promulgar el conocimiento y ostentar la verdad sobre la memoria de una comunidad. (Ojeda 2015, 34)

Estas instituciones se crearon como agentes discursivos del conocimiento que homogeneizaban e indicaban la última palabra frente a lo desconocido. ¿Será que los docentes contemplaban el poder de enunciación de las instituciones desde donde emanaba el conocimiento y pensaban en los intereses personales, regionales, y estatales que perseguían? Para establecer este análisis hay que ir más allá de la hermenéutica y pasar por un modelo de interpretación de la sociocrítica para poder comprender a la sociedad actual desde la historia.

Al revisar la introducción de La historia como arma de Manuel Moreno Fraginals (1984), en su estudio sobre esclavos, ingenios y plantaciones señala que la historia tiene un papel político por medio del cual se deben establecer los derroteros para justificar ciertos procedimientos del gobierno, en ese caso de la revolución cubana. Señalaba cómo la historia le servía para modelar un sujeto trabajador, comunitario y socialista. La historia entonces justificaba la revolución en contra de la explotación.

En palabras de Arendt, de la obra La condición humana:

Ser visto y oído por otros deriva su significado del hecho de que todos ven y oyen desde una posición diferente. Este es el significado de la vida pública, comparada con la cual incluso la más rica y satisfactoria vida familiar solo puede ofrecer la prolongación o multiplicación de la posición de uno con sus acompañantes aspectos y perspectivas. Solo donde las cosas pueden verse por muchos en una variedad de aspectos y sin cambiar su identidad, de manera que quienes se agrupan a su alrededor sepan que ven lo mismo en total diversidad, solo allí aparece auténtica y verdaderamente la realidad mundana. (Arendt 1958, 66)

De esta manera podemos señalar que la pluralidad no es simplemente el reconocimiento de la alteridad, sino que es el reconocimiento de la diversidad, pero más allá de esto es el reconocimiento de un espacio de deliberación. Por eso seguiremos el concepto de ámbito público a partir de Arendt quien contempla la participación ciudadana en un espacio de apariencias, de visibilidad, en el que tanto hombres y mujeres pueden ser vistos y oídos y revelar mediante la palabra y la 
acción cuáles son sus experiencias políticas y su participación ciudadana. (Arendt 2002, 232)

Solo la acción política, en el sentido de compartir y de una puesta en memoria de las proezas de los héroes, gracias a la capacidad de dar forma a un relato, a una historia, les permite a los hombres (sic) perdurar, no como especie, sino como pluralidad. (Kristeva, 1999, p 175

La historia es para Arendt el relato — story - de las acciones pasadas de los hombres. La acción pertenece a la dimensión de la vida activa de los hombres, que se opone a la vida contemplativa, junto a la labor y el trabajo. De este modo, aquí no se contempla la historia como objeto de estudio, ni la historia como proceso, siguiendo las filosofías de la historia como, por ejemplo, la lucha de clases, ni la historia procesual de la escuela de los Annales. Frente a esto Arendt afirmaba que:

Sólo él [Marx] comprendió que si se aceptaba que la historia es el objeto de un proceso de fabricación o del hacer, debe llegar un momento en que ese objeto esté terminado y en que, si se imagina que es posible hacer historia, no se puede ignorar la consecuencia de que la historia tendrá un fin (Arendt 1996, 89).

Las características de la condición humana para Arendt son la acción, la labor y el trabajo. Si la historia se concibe como una labor o un trabajo tendrá un producto terminado, pero en la acción se concibe la capacidad de creación y en esta medida se contempla la narración y las distintas formas de concebir el pasado y las distintas realidades.

En la acción el hombre desarrolla su capacidad de ser libre que lo distingue de los animales. La libertad no es la mera ausencia de coerción, sino que es la capacidad de introducir novedad en el mundo, es decir de producir efectos inesperados. (Arendt 1996)

Para Arendt, la acción es la actividad de creación de mayor jerarquía en el quehacer humano mientras que la labor se refiere a procesos vitales y cíclicos que dependen del organismo. La política y la acción tienen que ver con la libertad del ser humano. Por mucho que lo haya intentado negar, las filosofías de la historia, la inestabilidad y la fragilidad de las acciones hacen que la historia sea una "gran narración sin comienzo ni fin»(Arendt, 1993, 208). Desde allí se concibe la Historia Estelar. Distintas constelaciones y realidades enunciadas desde diferentes enfoques narrativos bajo círculos de interpretación o hermenéuticos. Entonces, la Historia Estelar contempla que los acontecimientos históricos hay que narrarlos en un contexto y lo que se pretende es comprender la acción humana de los sujetos, establecer las distintas experiencias políticas de la configuración social, para poder señalar en este proyecto la formación ciudadana desde la experiencia de cada uno en los distintos espacios de deliberación. 
De ese modo, dentro de la Historia Estelar propuesta aquí, lo que se quiere es establecer un modelo por medio del cual se pueda comprender la configuración de una sociedad en la construcción de ciudadanía con el fin de poder analizar la capacidad de acción y deliberación en la formación ciudadana. Por comprensión entendemos, de acuerdo a Hannah Arendt, un «fenómeno» distinto de la correcta información y del conocimiento científico, es un complicado proceso que nunca produce resultados inequívocos. Es una actividad sin fin, siempre diversa y mutable, por la que aceptamos la realidad. (Arendt 1998, 29)

La teórica de lo político, Arendt, en un artículo denominado Comprensión y política, señala que:

La historia [history] aparece cada vez que ocurre un acontecimiento lo suficientemente importante para iluminar su pasado. Entonces la masa caótica de sucesos pasados emerge como un relato [story] que puede ser contado, porque tiene un comienzo y un final (Arendt 1993, 29).

Es así que en la Historia Estelar, como modelo narrativo de uno o varios acontecimientos, se analiza la configuración social y las distintas experiencias políticas de los sujetos desde el prisma de la narración.

\section{Nuevo enfoque para enseñar la historia y dejar atrás la crisis del país}

Hoy en día el mismo sujeto es el que está produciendo su propio conocimiento. Ya no se necesita de los historiadores y de los científicos sociales para producir conocimiento pues el historiador da herramientas que transmite a la sociedad y el ciudadano produce su propio conocimiento; en fin, el historiador no es el único que produce conocimiento. En otras palabras, «un científico que sepa operar con lo plural y lo distinto, que sepa trabajar con la producción de textos, documentos y objetos provistos de significado» (Herrera 2009, 67) es lo que necesita la educación hoy. La enseñanza de la historia no es repetir memorísticamente el conocimiento, sino construir la comprensión de distintas interpretaciones del pasado para ponerlas en diálogo y respetar la pluralidad.

La sociedad debe consumir el pasado y debemos promoverlo. La gente debe identificarse con el pasado, la identidad debe ser producida por la ciudadanía. Los colombianos debemos tener identidad de nación. Esto no se ha logrado porque la historia ha sido impuesta, la gente no ha sido participe de un pasado, no han ayudado a construir su memoria, por eso cree que hay que hacer partícipe a las personas, ellos se deben sentir participes de un todo. 
Claves para la comprensión de una Historia Estelar: interpretación, comprensión, hermenéutica y diversidad política

Se debe también incentivar a que la sociedad produzca su propia memoria para que en el futuro los historiadores puedan encontrar en el archivo algunas fuentes que permitieran rastrear la vida cotidiana de la gente del común, por ejemplo, qué bueno sería que los profesores les permitieran a sus estudiantes recuperar la historia de sus abuelos, escribir diarios de sus familias en las que se consignen sus quehaceres y sus roles. Que esos diarios y esos cuadernos se puedan legar a las generaciones venideras y hasta depositar en las bibliotecas y archivos.

¿Qué pasaría si ya no se concibe la historia de la nación desde la élite? ¿Se tendrá que hablar sólo de la historia de la lucha armada o de la lucha de clases, como se ha pensado hasta ahora? ¿Se tendrá que hablar sólo de la historia de la abnegación o habrá posibilidad para hablar de la riqueza cultural de los otros?

Qué bueno promover en el aula de clase una crítica a los hitos históricos y proponer otros momentos en la historia como acontecimientos, otras miradas y otros actores. Promover la enseñanza de la historia que emancipa, que comprende y por ende perdona del pasado al presente. Una historia que promueva el Perdón como posibilidad política.

En el trabajo de Arendt se rescata un apartado que ilumina toda la investigación y es la historia como método de análisis desde la narración.

En contraposición a la fabricación, en la que la luz para juzgar el producto acabado la proporciona la imagen o modelo captado de antemano por el ojo del artesano, la luz que ilumina los procesos de la acción, y por tanto todos los procesos históricos, sólo aparece en su final, frecuentemente cuando han muerto todos los participantes. La acción sólo se revela plenamente al narrador, es decir, a la mirada del historiador, que siempre conoce mejor de lo que se trataba que los propios participantes. Aunque las historias son los resultados inevitables de la acción, no es el actor, sino el narrador, quien capta y hace la historia (Arendt 1998, 215).

Es así que en la historia pueden existir múltiples relatos o narraciones de los acontecimientos, la historia no tiene fin y por lo tanto no puede producir resultados definitivos (Arendt 1998, 30). Por esta razón, se propone aquí otra forma de reconstruir los hechos históricos no teniendo en cuenta una historia oficial, verdadera, sino una de tantas versiones o realidades de las acciones políticas de los sujetos que configuraron una sociedad en un tiempo determinado.

Arendt ya advertía el problema de la postmodernidad en los relatos y las narraciones históricas en las que todo vale, interpretaciones que se pudieron sacar desde la obra de Hayden White. El pasado no existe hasta que el narrador lo traiga al presente, pero es labor del historiador advertir su posición teórica y su 
intencionalidad. «Aun si se admite que cada generación tiene derecho a escribir su propia historia, sólo le reconocemos el derecho de acomodar los acontecimientos según su propia perspectiva, pero no el de alterar la materia objetiva misma» (Arendt, 1996:251).

\section{Conclusiones}

El objeto de estudio de la historia reconoce la interdisciplinariedad con áreas como la política, la economía, la sociedad y la cultura, entendidas como puntos de referencia que permiten profundizar en el estudio de la historia. Sin embargo, «entendemos que la historia tiene como objeto principal el estudio del hombre en el pasado y no simplemente la búsqueda y organización de hechos» (Ricoeur 2004, 222-223). Dios creó al ser humano, hombre y mujer, y allí se contempló una relación política de deliberación, es decir, se generó un espacio de reflexión en los seres humanos, de comprensión y de reconstrucción, pero al mismo tiempo de la acción.

Lo más importante de este objeto de estudio es la humanidad interpretada desde dos variables imprescindibles: el tiempo y el espacio. Sin éstas no se podrán ubicar las acciones pasadas de los seres humanos ni estudiar su legado para las sociedades presentes y futuras, por tal motivo es conveniente que el historiador establezca un diálogo con sus pares de otras disciplinas, en estos aspectos, para contribuir al desarrollo de la investigación.

Es así que la formación de ciudadanos a partir de la historia posibilita, más allá de los recursos materiales en general y de cada uno de los aspectos en particular que se han tocado aquí, el desarrollo de proyectos de investigación en torno a la reconstrucción de la memoria y la identidad nacional, pero más allá de esto, la búsqueda y solución a problemas de la realidad social desde distintos campos del conocimiento, entre ellos, la historia.

Por tal motivo me gustaría proponer aquí otra mirada a nuestro pasado, no solo desde la violencia en Colombia o la imposición de una identidad nacional como historia oficial, sino tomando, como en el cuento de Blancanieves la versión de la Bruja malvada, la de los enanos, el Príncipe azul y, sobre todo, la versión que posibilita, más que la totalidad, la inclusión de los actores, un enfoque constructivo de la identidad nacional que tanto necesita este país. Una Historia Estelar. 


\section{Referencias}

Archila, M. La disciplina histórica en la Universidad Naciona. Bogotá: UNAL, 1997.

Arendt, H. (1961) Entre el pasado y el futuro. ocho ejercicios sobre la reflexión política. Barcelona: Península.

—. (1953) De la historia a la acción. Barcelona: Paidos.

-. (1993) La condición humana. Barcelona: Paidós.

Barbosa, M. (2001) "Medios de comunicación y conmemoraciones. Estrategias de reactualización y construcción de la memoria." Signo y Pensamiento: 104-112.

Benjamin, W. (2001) Iluminaciones II. Poesía y capitalismo. Madrid: Taurus.

Bernabeud, S. (1987) 1892, el IV centenario del descubrimiento de América. Madrid: Consejo Superior de Investigaciones Científicas.

Bonilla, H. (1997) "Diseño curricular de una Licenciatura en Historia con énfasis en la Historia de la América Latina." Revista Universidad del Atlántico: 7-15.

Collingwood, R. (2004) Idea de la Historia. México: Fondo de Cultura Económica.

Febvre, L. (1982) Combates por la historia. Barcelona: Ariel.

—. (1959) El problema de la incredulidad en el siglo XVI. La religión de Rabelais. Mexico: UTAH.

Henao, J.M., Arrubla, G. (1910) Historia de Colombia. Bogotá: Academia de Historia de Colombia.

Herrera, J. (2009) La comprensión de lo social. Horizonte hermeneutico de las ciencias sociales. Bogotá: Cinde.

Le Goff, J. (1991) Pensar la historia. Barcelona: Paidos.

Lewis, J. (2006) La inmovilidad de los textos. La nación en los manuales de Historia de Colombia, 1911-1957. Bogotá: CESO, Universidad de Los Andes.

Proust, M. (2000) En busca del tiempo perdido. Madrid: Alianza.

Melo, J.O. (1999). "Medio siglo de historia colombiana: notas para un relato inicial." Revista de Estudios Sociales: 9-22.

Ojeda,, R. (2005). Fuentes documentales para el estudio de las haciendas en Ubaté. La hacienda el Hato de Subia. Bogotá: Gimnasio Campestre.

—. (2007) "La paleografía como herramienta que posibilita la comprensión de la historia colonial." Revista El Astrolabio: 25-38.

—. (2015) "Memoria, historia y ruralidad: teoría y métodos." In Las nuevas miradas de la comprensión de la historia y la memoria, by Sebastian (ed.) González. Bogotá: Universidad de La Salle.

Pereña, L. (1990) Descubrimiento y conquista, ¿genocidio? Salamanca: Universidad Pontificia de Salamanca. 
Ricoeur, P. (2003) La memoria, la historia, el olvido. Madrid: Trotta.

Rodriguez, M. (1982) "El 12 de octubre: entre el IV y el V centenario." In Cultura e identidad nacional, by Roberto (comp.) Blancarte. México: Fondo de Cultura Económica.

Siciliani, J.M. (s/f) Una mirada interrogativa al Doctorado en Educación y Sociedad desde el recorrido de la hermenéutica contemporánea. Seminario de Fundamentación Teórica. Doctorado.

Silva, R. (2003) "La servidumbre de las fuentes." In Balance y desafío de la historia de Colombia al inicio del siglo XXI, by Adriana Maya and Diana Bonnet. Bogotá: Universidad de Los Andes.

Todorov, T. (2000) Los abusos de la memoria. Barcelona: Paidos.

Wills, M.E. (1999) "De la nación católica a la nación multicultural: rupturas y desafíos." Museo, memoria y nación: 387-411.

Zapata, M.I. (2004) "Walter Benjamin, historia cultural y fotografía." Memoria y Socieda: 5-16.

Zorraquin, R. (1992) Dos continentes y una sola cultura. Buenos Aires, México: Academia Nacional de Historia, Universidad de Guadalajara.

Recibido: 19 de enero 2015

Aceptado: 6 de mayo 2015

Cómo citar: Ojeda, R. (2015) Claves para la comprensión de una

Historia Estelar: interpretación, comprensión, hermenéutica y

diversidad política. Praxis Pedagógica, 69-84 